\title{
Bevölkerungseinstellungen zur Demokratie: Kein Grundkonsens zwischen Ost- und Westdeutschen
}

\author{
Oskar Niedermayer
}

In der Demokratieforschung besteht grundsätzliche Übereinstimmung darüber, dass aus normativer Sicht ein deutlicher Mangel an Unterstützung der demokratischen politischen Ordnung durch die Bevölkerung - also eine Inkongruenz von politischer Struktur und politischer Kultur - dem Selbstverständnis der Demokratie als einer durch die Zustimmung der Bürger legitimierten Herrschaftsordnung widerspricht. Über das konkrete Ausmaß an positiven Einstellungen zur politischen Ordnung, das einer Demokratie angemessen ist, gibt es allerdings keinen Konsens. ${ }^{1}$ Die Beurteilung wird zudem dadurch erschwert, dass es für eine umfassende Analyse der Einstellungen der Bürger gegenüber der demokratischen politischen Ordnung notwendig ist, verschiedene Objektebenen zu unterscheiden.

Bettina Westle differenziert in ihrer Weiterentwicklung des Konzeptes der politischen Unterstützung von David Easton ${ }^{2}$ vier solcher Objektebenen: die politische Philosophie als Grundlage des Gemeinwesens, unterschiedliche Interpretationen der Philosophie in Form von politischen Ordnungsvorstellungen, die formale politische Ordnung, das heißt die in einer Verfassung konkretisierte Interpretation der politischen Philosophie, und die materiale politische Ordnung, das heißt die Verfassungswirklichkeit. ${ }^{3}$ Ein etwas vereinfachtes Modell mit drei Objektebenen schlägt Dieter Fuchs vor. Er unterscheidet die Werte-, Strukturund Performanzebene ${ }^{4}$ : (1) Die Werteebene bezieht sich auf die Idee der Demokratie selbst als normatives Ordnungsmodell und auf normative Vorstellungen darüber, wie eine Demokratie im Einzelnen aussehen sollte. Angesichts der Vielfalt normativer Prinzipien, die mit dem Demokratiebegriff verbunden werden, können diese Vorstellungen sehr unterschiedlich ausfallen; sie lassen sich jedoch zu einer begrenzten Zahl von normativen Modellen der Demokratie zusammenfassen. ${ }^{5}$ (2) Die Strukturebene bezieht sich auf die in der Regel durch eine Verfassung implementierte Ausformung der demokratischen Ordnung eines Landes, also in Deutschland auf die durch das Grundgesetz festgelegte spezifische Form von Demokratie. (3) Die Performanzebene bezieht sich auf den alltäglichen demokratischen Prozess, auf das Funktionieren der Demokratie und ihren aktuellen Zustand, also auf die Verfassungswirklichkeit.

1 An der Feststellung Arend Lijpharts Ende der 1980er Jahre, es bestünde keine Klarheit darüber, welche Verteilung welcher konkreten Einstellungen eine demokratische politische Kultur ausmacht, hat sich bis heute nichts geändert; vgl. Arend Lijphart, The Structure of Inference, in: Gabriel A. Almond I Sidney Verba (Hrsg.), The Civic Culture Revisited, Newbury Park u.a. 1989, S. 37 - 56.

2 Vgl. David Easton, A Systems Analysis of Political Life, New York 1965 und ders., A Re-assessment of the Concept of Political Support, in: British Journal of Political Science, 5. Jg. (1975), H. 4, S. $435-457$.

3 Vgl. Bettina Westle, Politische Legitimität - Theorien, Konzepte, empirische Befunde, Baden-Baden 1989, S. $170 \mathrm{ff}$.

4 Vgl. Dieter Fuchs, Welche Demokratie wollen die Deutschen? Einstellungen zur Demokratie im vereinten Deutschland, in: Oscar W. Gabriel (Hrsg.), Politische Orientierungen und Verhaltensweisen im vereinigten Deutschland, Opladen 1997, S. 81 - 114, S. 83 ff.

5 Dieter Fuchs unterscheidet ein libertäres Modell, ein liberales Modell, ein Modell des demokratischen Sozialismus und ein republikanisches Modell; vgl. Dieter Fuchs, a.a.O. (Fn. 4), S. 86 ff. 
Für ein Auseinanderfallen von Verfassungsnorm und -wirklichkeit lassen sich verschiedene Gründe denken: Erstens verhält sich das politische Führungspersonal immer nur mehr oder weniger rollen- und normenkonform; zweitens können die politischen Institutionen anders funktionieren als durch das Grundgesetz vorgesehen; und drittens wird die Beurteilung der Verfassungswirklichkeit auch von nichtpolitischen - zum Beispiel von ökonomischen Entwicklungen beeinflusst.

Übernimmt man diese Einteilung und bezieht alle drei Objektebenen in die Analyse ein, so lässt sich eine Typologie der Einstellungen zur demokratischen politischen Ordnung bilden, die vier Typen unterscheidet ${ }^{6}$ :

- zufriedene Demokraten, die der Idee der Demokratie positiv gegenüberstehen und sowohl mit der im Grundgesetz festgelegten spezifischen Form der Demokratie als auch mit ihrem tatsächlichen Funktionieren zufrieden sind;

- politikkritische Demokraten, die der Idee der Demokratie positiv gegenüberstehen und mit der im Grundgesetz festgelegten Form der Demokratie zufrieden, mit dem tatsächlichen Funktionieren der Demokratie jedoch unzufrieden sind;

- systemkritische Demokraten, die der Idee der Demokratie positiv gegenüberstehen, aber sowohl mit der im Grundgesetz festgelegten spezifischen Form der Demokratie als auch mit ihrem tatsächlichen Funktionieren unzufrieden sind;

- Antidemokraten, die sowohl mit dem tatsächlichen Funktionieren der Demokratie in Deutschland als auch mit der im Grundgesetz festgelegten spezifischen Form der Demokratie unzufrieden sind und auch von der Demokratie als Idee nichts halten.

Für die Bundesrepublik vor der Wiedervereinigung kommen zahlreiche empirische Studien zu dem Ergebnis, dass sich nach und nach ein auf Idee, konkrete Form und Praxis der Demokratie bezogener demokratischer Grundkonsens entwickelt hatte ${ }^{7}$, der im Vergleich mit der Bevölkerung anderer demokratischer Staaten überdurchschnittlich stark ausgeprägt war, so dass die alte Bundesrepublik in den 1980er Jahren ,als Musterbeispiel für ein positives Verhältnis der Bevölkerung zum politischen System" "8 galt. Nach der Wiedervereinigung erhält die Untersuchung der Bevölkerungseinstellungen zur Demokratie neben der Frage nach der Legitimation der politischen Ordnung durch die Zustimmung der Bürger im gesamten Deutschland zusätzliche Relevanz durch die Frage, ob der Transfer der politischen Ordnung der alten Bundesrepublik auf Gesamtdeutschland im Osten der Republik von einem komplementären Wandel der Bevölkerungseinstellungen begleitet wurde, oder ob wir es in diesem Bereich auch heute noch mit zwei unterschiedlichen politischen Kulturen

6 Vgl. Oskar Niedermayer, Bürger und Politik. Politische Orientierungen und Verhaltensweisen der Deutschen, Wiesbaden 2005, S. 107.

7 Vgl. zum Beispiel Kendall L. Baker / Russell J. Dalton / Kai Hildebrandt, Germany Transformed: Political Culture and the New Politics, Cambridge 1981; David P. Conradt, Changing German Political Culture, in: Gabriel A. Almond / Sidney Verba (Hrsg.), The Civic Culture Revisited, Boston 1980, S. 212 - 272; Dieter Fuchs, Die Unterstützung des politischen Systems der Bundesrepublik Deutschland, Opladen 1989; Oscar W. Gabriel, Demokratiezufriedenheit und demokratische Einstellungen in der Bundesrepublik Deutschland, in: APuZ, B 22 (1987), S. 32 - 45; Robert Rohrschneider, Learning Democracy. Democratic and Economic Values in Unified Germany, Oxford 1999.

8 Oscar W. Gabriel, Politische Einstellungen und politische Kultur, in: Oscar W. Gabriel / Sabine Kropp (Hrsg.), Die EU-Staaten im Vergleich, Wiesbaden 2008, S. 181 - 214, S. 193; vgl. auch Peter H. Merkl, Comparing Legitimacy and Values Among Advanced Industrial Countries, in: Mattei Dogan (Hrsg.), Comparing Pluralist Democracies, Boulder 1988, S. 19 - 63. 
zu tun haben. Die folgende empirische Analyse der Entwicklung der Bevölkerungseinstellungen zur Demokratie ${ }^{9}$, die zunächst die Werte-, Struktur- und Leistungsebene getrennt betrachtet und danach in der oben beschriebenen Typologie zusammenfügt, richtet daher ihr besonderes Augenmerk auf mögliche Einstellungsunterschiede zwischen West- und Ostdeutschen.

\section{Die Entwicklung der Einstellungen zur Demokratie als Staatsidee und zu den normativen Prinzipien der Demokratie}

In allen hier analysierten Umfragen bekundet die große Mehrheit der Deutschen ihre Zustimmung zur Idee der Demokratie als politisches Ordnungsmodell. Mitte 2008 sprachen sich 92 Prozent der gesamtdeutschen Bevölkerung dafür aus und nur fünf Prozent dagegen (vgl. Tabelle 1). In der Intensität der Befürwortung zeigen sich jedoch signifikante Unterschiede: Während in Westdeutschland eine deutliche absolute Mehrheit von 59 Prozent „sehr“ für die Idee der Demokratie ist, zählt in Ostdeutschland nur die relative Mehrheit von 44 Prozent zu den uneingeschränkten Befürwortern. Diese Differenz erscheint in etwas günstigerem Licht, wenn diejenigen hinzugezählt werden, die „ziemlich für die Idee der Demokratie“ sind, denn dann stehen 83 Prozent der Westdeutschen immerhin 74 Prozent der Ostdeutschen gegenüber.

Das hohe generelle Niveau an Zustimmung zur Demokratie als Ordnungsmodell in beiden Landesteilen und die signifikanten Ost-West-Unterschiede in der Intensität der Befürwortung existierten von Anfang an, wie Abbildung 1 anhand der Mittelwerte der Antworten auf diese Frage verdeutlicht. ${ }^{10}$ Die leichten Schwankungen ${ }^{11}$ im Zeitverlauf lassen

9 Die dabei verwendeten Daten von 2002 bis 2008 stammen aus Studien, deren Daten dem Verfasser zur Verfügung stehen. Die repräsentativen Bevölkerungsumfragen dieser Forschungsprojekte weisen unterschiedliche Altersgrenzen auf. Für die Analysen wurden hier aus Vergleichbarkeitsgründen nur die Daten für Befragte ab 18 Jahren verwendet. Im Einzelnen handelt es sich um die folgenden Studien: 2002: „Rechtsextreme Einstellungen in Deutschland“ (Projektleiter: Elmar Brähler, Finanzierung: Universität Leipzig, N = 2025); 2003: „Gewerkschaften und Rechtsextremismus" (Projektleiter: Bodo Zeuner, Michael Fichter und Richard Stöss, Finanzierung: HansBöckler-Stiftung, N = 3928); 2005: „Bürger und Parteien“ (Projektleiter: Steffen Kühnel, Oskar Niedermayer und Bettina Westle, Finanzierung: DFG, N = 2540); 2008: „Demokratische und rechtsextreme Bevölkerungseinstellungen“ (Projektleiter: Oskar Niedermayer und Richard Stöss; Finanzierung: Deutsche Paul Lazarsfeld-Gesellschaft, N = 5681), Berlin-Brandenburg-Bus 2008 (Projektleiter: Oskar Niedermayer und Richard Stöss; Finanzierung: Deutsche Paul Lazarsfeld-Gesellschaft, $N=1940$ ). Zu den Ergebnissen der letzten beiden Studien für die Gesamtheit der Befragten ab 14 Jahren, die nur geringfügig von den hier berichteten Ergebnissen abweichen, vgl. Oskar Niedermayer / Richard Stöss, Einstellungen zur Demokratie in Berlin und Brandenburg 2002-2008 sowie in Gesamtdeutschland 2008, Berlin 2008.

10 Mittelwerte auf der Basis einer 6-Punkte-Skala von $-1=$ „sehr gegen die Idee der Demokratie“ bis $+1=$,sehr für die Idee der Demokratie“. Eigene Berechnungen, Ausgangsdaten 1985 bis 1993 aus Bettina Westle, Demokratie und Sozialismus. Politische Ordnungsvorstellungen im vereinten Deutschland zwischen Ideologie, Protest und Nostalgie, in: Kölner Zeitschrift für Soziologie, 46. Jg. (1994), H. 4, S. 571 - 596, S. 580. Für 2002 bis 2008 stammen die Daten aus den in Fußnote 9 angegebenen Studien.

$11 \mathrm{Zu}$ beachten ist, dass die größten Schwankungen zweier aufeinanderfolgender Werte nicht viel mehr als fünf Prozent der gesamten Skalenbreite ausmachen. 
einen gewissen Einfluss von politischen Ereignissen auch auf die generalisierte Unterstützung der politischen Ordnung vermuten. So zeigt sich zum Beispiel die stärkste Unterstützung zum Zeitpunkt der mit hohen Erwartungen seitens der Bevölkerung verbundenen Bildung der Großen Koalition Ende 2005.

\section{Tabelle 1: Einstellungen zur Demokratie als Staatsidee (2008, Angaben in Prozent)}

„Die drei folgenden Fragen beschäftigen sich mit der Demokratie. Zunächst geht es nicht um tatsächlich bestehende Demokratien, sondern um die Idee der Demokratie. Was würden Sie, im Vergleich zu anderen Staatsideen, zur Idee der Demokratie sagen. Sind Sie ...?"

\begin{tabular}{|l|ccc|}
\hline & Gesamt & Ost & West \\
\hline Sehr für die Idee der Demokratie & 56 & 44 & 59 \\
Ziemlich für die Idee der Demokratie & 25 & 30 & 24 \\
Etwas für die Idee der Demokratie & 11 & 15 & 10 \\
Etwas gegen die Idee der Demokratie & 3 & 3 & 3 \\
Ziemlich gegen die Idee der Demokratie & 1 & 2 & 1 \\
Sehr gegen die Idee der Demokratie & 1 & 2 & 1 \\
\hline
\end{tabular}

Zu 100 Prozent fehlende Werte: weiß nicht / keine Antwort; $N=5681$.

Quelle: DPLG-Projekt „Demokratische und rechtsextreme Bevölkerungseinstellungen“, a.a.O. (Fn. 9).

In unseren Daten bleibt der Abstand zwischen Ost und West über die Zeit hinweg in etwa gleich und ein ausgeprägter Trend ist nicht zu erkennen, während andere Untersuchungen mit dem gleichen Fragewortlaut, aber unterschiedlicher Antwortvorgabe zu dem Ergebnis kommen, dass sich die Ost-West-Differenz 1998 und 2002 deutlich vergrößert hat. ${ }^{12}$ Eindeutig ist somit davon auszugehen, dass die zunehmenden eigenen Erfahrungen der Ostdeutschen mit der liberal-pluralistischen Demokratie bisher nicht zu einer Verstärkung der generalisierten Systemunterstützung und einer Konvergenz der Orientierungen in Ost und West geführt haben.

Betrachtet man statt der generellen Unterstützung der Idee der Demokratie die Befürwortung einzelner normativer Prinzipien, die eine Demokratie ausmachen, so zeigte die erste detaillierte empirische Studie Ende der 1960er Jahre ${ }^{13}$, dass unter den Bürgern der alten Bundesrepublik über abstrakte demokratische Prinzipien wie das Recht auf freie Meinungsäußerung, die Möglichkeit einer alternierenden Parteienregierung und die Notwendigkeit einer politischen Opposition praktisch Konsens bestand und auch das Demonstrationsrecht und der Gewaltverzicht bei der politischen Konfliktaustragung noch breite Zustimmung fanden. Andere Merkmale einer demokratischen Regierungsform wie die

12 Vgl. Oscar W. Gabriel, Politische Einstellungen und politische Kultur, in: Oscar W. Gabriel / Everhard Holtmann (Hrsg.), Handbuch politisches System der Bundesrepublik Deutschland, München 2005, S. 457 - 522, S. 491 und Kerstin Völkl, Fest verankert oder ohne Halt? Die Unterstützung der Demokratie im vereinigten Deutschland, in: Oscar W. Gabriel / Jürgen W. Falter / Hans Rattinger (Hrsg.), Wächst zusammen, was zusammengehört?, Baden-Baden 2005, S. 249 284, S. 254. Die Antwortvorgabe dort lautete: „Die Zahl ,1' bedeutet, dass die Demokratie die beste vorstellbare Staatsidee ist, die Zahl ,6' bedeutet, dass die Demokratie die schlechteste vorstellbare Staatsidee ist".

13 Vgl. Max Kaase, Demokratische Einstellungen in der Bundesrepublik Deutschland, in: Rudolf Wildenmann u.a. (Hrsg.), Sozialwissenschaftliches Jahrbuch für Politik, Bd. 2, München 1971, S. $119-326$. 


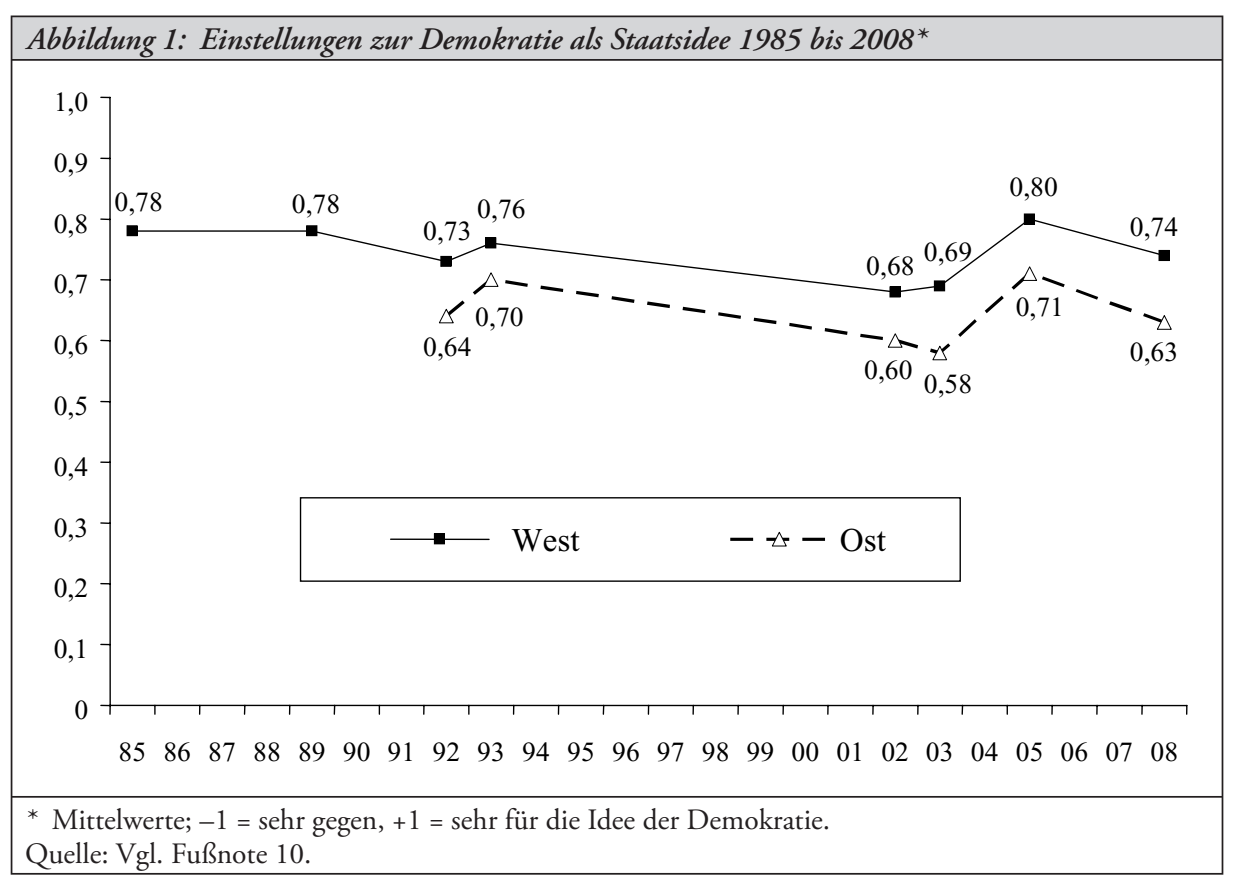

Aufgabe der Opposition, die Regierung zu kritisieren, die Befürwortung von Interessenpluralismus und das Primat der Meinungsfreiheit gegenüber der öffentlichen Ordnung waren jedoch stark umstritten beziehungsweise wurden sogar mehrheitlich abgelehnt. In der Folgezeit ließen „alle seit 1979 durchgeführten Untersuchungen ... die bereits 1968 festgestellte Spannung zwischen einer breiten Akzeptanz allgemeiner demokratischer Grundwerte und einer überwiegend negativen Einstellung zur Rolle von Konflikt, Kritik und Wettbewerb in der Demokratie erkennen "14. Zunächst wurde dies im Sinne des Fortwirkens obrigkeitsstaatlicher Traditionen interpretiert. Diese Position ist allerdings problematisch, „weil sie ein linksliberales Demokratieverständnis mit demokratischen Überzeugungen gleichsetzt" ${ }^{15}$. Die Tatsache, dass die Orientierungen gegenüber allgemeinen demokratischen Prinzipien und die Bewertung von Konflikt weitgehend unabhängig voneinander sind $^{16}$, deutet darauf hin, „dass bestimmte Bevölkerungsgruppen in Deutschland ein konfliktäres, andere aber ein konsensorientiertes Demokratiemodell favorisieren. Beide stehen aber - wenn auch mit divergierenden Vorstellungen - auf dem Boden der Demokratie“17.

Für Ostdeutschland war kurz nach der Wende nicht mit einer ähnlich breiten Zustimmung zu den einzelnen demokratischen Prinzipien und Werten zu rechnen wie in Westdeutschland, da die DDR demokratische Werte der herrschenden Staatsdoktrin des demokratischen Sozialismus angepasst und sich von der bürgerlich-liberalen Demokratie des

14 Oscar W. Gabriel, a.a.O. (Fn. 12), S. 491.

15 Ebenda, S. 492.

16 Vgl. hierzu Bettina Westle, Politische Folge- und Kritikbereitschaft der Deutschen, in: ZUMANachrichten, 21. Jg. (1997), H. 2 (Nummer 41), S. 100 - 126.

17 Oscar W. Gabriel, a.a.O. (Fn. 12), S. 492. 
Westens deutlich abgegrenzt hatte. Umso erstaunlicher waren die Ergebnisse der ersten, noch in der DDR durchgeführten Umfrage zu diesem Bereich ${ }^{18}$, nach denen sich die demokratischen Orientierungen in West- und Ostdeutschland nur graduell unterschieden.

Zur Erklärung dieser Tatsache wurden mehrere Hypothesen angeboten ${ }^{19}$ : Die während der Zeit der Teilung fortbestehenden Kommunikationsbeziehungen zwischen Ost und West könnten die DDR-Bevölkerung zu einer Übernahme westlicher Ordnungsvorstellungen veranlasst haben; die demokratische Revolution in der DDR könnte zu jenen einschneidenden Ereignissen in der Geschichte eines Volkes gehört haben, die einen dramatischen, längerfristigen Wandel politischer Wertorientierungen mit sich bringen; oder die im Wertesystem des Sozialismus angelegten demokratischen Potenziale könnten durch die repressive Praxis des DDR-Regimes nicht gänzlich neutralisiert worden sein und sich daher nach dem Kollaps dieses Systems wieder Geltung verschafft haben. ${ }^{20}$ Wegen des Fehlens verlässlicher Erkenntnisse über die Erfolge der DDR-Sozialisation und mangelnder Umfragedaten aus der DDR-Zeit lassen sich die Gründe heute allerdings nicht mehr eindeutig feststellen.

Alle diese Erklärungsversuche und die Tatsache, dass sich hinsichtlich der Unterstützung der Meinungsfreiheit, des Rechts auf Opposition und alternierender Parteienregierung auch in der Folgezeit kaum ein Ost-West-Unterschied zeigte ${ }^{21}$, implizieren, „dass sich bereits kurz nach der Vereinigung ein demokratischer Grundkonsens zwischen den Ost- und den Westdeutschen entwickelt“ hat. „Allerdings gibt es auch Anhaltspunkte für eine weniger optimistische Interpretation. "22 Zum einen zeigen die Ergebnisse einer Untersuchung der Stabilität und Kohärenz demokratischer Orientierungen der Ost- und Westdeutschen ${ }^{23}$, dass „das Verhältnis der Ostdeutschen zur Demokratie ... weniger gefestigt und weniger widerspruchsfrei ist als das ihrer Landsleute im Westen “24. Zum anderen deutet Vieles darauf hin, dass die Bürger in Ost- und Westdeutschland mit dem Demokratiebegriff unterschiedliche Vorstellungen verbinden. Eine Untersuchung des Demokratieverständnisses in Ost und West kurz nach der Vereinigung 25 zeigte, dass die Westdeutschen ein liberales beziehungsweise sozialdemokratisches Demokratiemodell befürworteten, während in Ostdeutschland die Synthese von Demokratie und Sozialismus zahlreiche Anhänger fand. Vor

18 Vgl. Petra Bauer, Freiheit und Demokratie in der Wahrnehmung der Bürger in der Bundesrepublik und der ehemaligen DDR, in: Rudolf Wildenmann (Hrsg.), Nation und Demokratie. Politisch-strukturelle Gestaltungsprobleme im neuen Deutschland, Baden-Baden 1991, S. 99 - 124.

19 Vgl. Russell J. Dalton, Communists and Democrats: Democratic Attitudes in the Two Germanies, in: British Journal of Political Science, 24. Jg. (1994), H. 4, S. 469 - 493 und Oscar W. Gabriel, Ein demokratischer Konsens in Ost und West?, in: Der Bürger im Staat, 50. Jg. (2000), H. 4, S. $196-202$.

20 Für die letzte Argumentation spricht zum Beispiel, dass für die ostdeutsche Bevölkerung eine Befürwortung der Idee des Sozialismus keine Abwendung von der Idee der Demokratie einschloss: 60 bis 70 Prozent befürworteten gleichzeitig beide Ordnungsideen, vgl. Bettina Westle, a.a.O. (Fn. 10).

21 Vgl. Kerstin Völkl, a.a.O. (Fn. 12), S. 254 f.

22 Oscar W. Gabriel, a.a.O. (Fn. 12), S. 493 f.

23 Vgl. Oscar W. Gabriel, Demokratische Einstellungen in einem Land ohne demokratische Traditionen? Die Unterstützung der Demokratie in den neuen Bundesländern im Ost-West-Vergleich, in: Jürgen W. Falter / Oscar W. Gabriel / Hans Rattinger (Hrsg.), Wirklich ein Volk? Die politischen Orientierungen von Ost- und Westdeutschen im Vergleich, Opladen 2000, S. 41 - 77.

24 Oscar W. Gabriel, a.a.O. (Fn. 12), S. 494.

25 Vgl. Dieter Fuchs, a.a.O. (Fn. 4). 


\begin{tabular}{|c|c|c|c|c|c|c|c|c|c|c|}
\hline \multicolumn{11}{|c|}{$\begin{array}{l}\text { Tabelle 2: Zuordnung normativer Prinzipien zur Demokratie (1978 bis 2000, } \\
\text { Angaben in Prozent; Mehrfachnennungen) }\end{array}$} \\
\hline \multicolumn{11}{|c|}{$\begin{array}{l}\text { "Was ist Ihrer Meinung nach das Wichtigste an der Demokratie, was von dieser Liste gehört unbedingt dazu, } \\
\text { dass man von einem Land sagen kann: Das ist eine Demokratie?" }\end{array}$} \\
\hline & \multirow{2}{*}{$\begin{array}{c}1978 \\
\mathrm{~W}\end{array}$} & \multirow{2}{*}{$\begin{array}{c}1986 \\
\text { W }\end{array}$} & \multicolumn{2}{|c|}{1990} & \multicolumn{2}{|c|}{1991} & \multicolumn{2}{|c|}{1993} & \multicolumn{2}{|c|}{2000} \\
\hline & & & $\mathrm{O}$ & W & $\mathrm{O}$ & W & $\mathrm{O}$ & W & $\mathrm{O}$ & W \\
\hline Liberale Grundrechte (MW) & 80 & 79 & 80 & 81 & 60 & 73 & 65 & 77 & 55 & 73 \\
\hline Presse und Meinungsfreiheit & 85 & 89 & 92 & 91 & 83 & 89 & 83 & 90 & 72 & 87 \\
\hline Religionsfreiheit & 78 & 73 & 83 & 79 & 60 & 71 & 66 & 75 & 54 & 67 \\
\hline Reisefreiheit & 79 & 79 & 76 & 78 & 53 & 69 & 60 & 76 & 53 & 70 \\
\hline Freie Berufswahl & 78 & 73 & 67 & 75 & 44 & 64 & 50 & 66 & 40 & 66 \\
\hline Pluralistischer Parteienwettbewerb (MW) & 73 & 76 & 80 & 78 & 66 & 78 & 71 & 77 & 55 & 69 \\
\hline Mehrere Parteien & 79 & 84 & 85 & 87 & 70 & 85 & 77 & 86 & 62 & 80 \\
\hline Freie und geheime Wahlen & 74 & 80 & 84 & 83 & 66 & 82 & 75 & 83 & 60 & 73 \\
\hline Regierungskontrolle durch starke Opposition & 67 & 65 & 70 & 65 & 62 & 66 & 62 & 61 & 43 & 55 \\
\hline \multicolumn{11}{|l|}{ Rechtsstaat } \\
\hline Unabhängige Gerichte & 68 & 74 & 76 & 74 & 62 & 77 & 66 & 76 & 51 & 66 \\
\hline Umfass. u. direkte Bürgerbeteiligung (MW) & 54 & 56 & 72 & 56 & 62 & 57 & 66 & 60 & 47 & 51 \\
\hline Bürgermitwirkung an vielen Entscheidungen & 51 & 56 & 68 & 55 & 57 & 59 & 67 & 66 & 47 & 54 \\
\hline Volksabstimmungen bei wichtigen Fragen & 56 & 55 & 75 & 52 & 68 & 57 & 72 & 60 & 55 & 56 \\
\hline Mitbestimmung in den Betrieben & 54 & 57 & 73 & 60 & 62 & 54 & 59 & 53 & 38 & 44 \\
\hline Soziale Gerechtigkeit (MW) & 49 & 44 & 58 & 36 & 53 & 36 & 51 & 40 & 39 & 32 \\
\hline Niemand muss Not leiden & 61 & 55 & 72 & 50 & 61 & 45 & 59 & 51 & 48 & 42 \\
\hline Begrenzte Einkommensunterschiede & 36 & 32 & 44 & 22 & 44 & 26 & 42 & 29 & 29 & 21 \\
\hline
\end{tabular}

die Wahl zwischen den beiden Grundwerten Freiheit und Gleichheit gestellt, sprachen sich die Westdeutschen mehrheitlich für die Freiheit, die Ostdeutschen dagegen für die Gleichheit aus. Zudem sahen die ostdeutschen Bürger auch einen größeren Bedarf an Verfassungsänderungen in Richtung einer Stärkung des Sozialstaates und der direkten Demokratie.

Für die Mehrheit der Ostdeutschen besitzt der Wert der Gleichheit nach wie vor Priorität vor dem Wert der Freiheit, während für die Westdeutschen das Gegenteil gilt. ${ }^{26}$ Zudem entwickelten sich die im Jahr der Vereinigung noch in vielen Bereichen identischen Vorstellungen der Ost- und Westdeutschen über essenzielle Merkmale einer Demokratie schon kurz danach auseinander und hatten sich im Jahre 2000 - neuere Daten stehen nicht zur Verfügung - noch nicht wieder angeglichen (vgl. Tabelle 2).

Ein Jahrzehnt nach der Vereinigung sahen die Westdeutschen die liberalen Grundrechte der Presse-, Meinungs-, Religions- und Reisefreiheit sowie der freien Berufswahl in deutlich höherem Maße als die Ostdeutschen als unverzichtbares Merkmal einer Demokratie an. Dasselbe gilt für die Prinzipien des pluralistischen Parteienwettbewerbs in Form der Existenz mehrerer Parteien, der Garantie freier und geheimer Wahlen und der Kontrolle der 
Regierung durch eine starke Opposition sowie für das rechtsstaatliche Prinzip unabhängiger, nur nach den Gesetzen urteilender Gerichte. Selbst bei den verschiedenen Formen umfassender und direkter Bürgerbeteiligung (der Mitwirkung der Bürger an politischen Entscheidungen, der Existenz von Volksabstimmungen und der betrieblichen Mitbestimmung), die 1990 in Ostdeutschland im Vergleich zum Westen noch stärker als unverzichtbares Element einer Demokratie angesehen wurden, sind die Zustimmungsraten im Jahre 2000 bei den Ostdeutschen geringer als bei den Westdeutschen. Ein starker Sozialstaat, in dem bei begrenzten Einkommensunterschieden niemand Not leidet, wird dagegen auch im Jahre 2000 im Osten noch stärker als unverzichtbares Merkmal der Demokratie angesehen als im Westen.

Betrachtet man die Entwicklung der mittleren Zustimmungswerte von 1990 bis 2000, so wird deutlich, dass das Zustimmungsniveau in allen Bereichen nicht nur in Ostdeutschland, sondern auch in Westdeutschland gesunken ist, während dort bis zur Vereinigung eine weitgehend konstante Unterstützung der essenziellen Demokratiemerkmale festzustellen war. Allerdings war der Zustimmungsrückgang im Osten sehr viel stärker und führte so zu einem Auseinanderklaffen der Orientierungsmuster.

Diese empirischen Befunde zur Entwicklung der verschiedenen Indikatoren generalisierter Systemunterstützung sind somit - vor allem für Ostdeutschland - nicht frei von einer gewissen Ambivalenz. Auf jeden Fall kann jedoch festgestellt werden, dass ein im gesamten Deutschland hohes Niveau der Unterstützung der Demokratie und ihrer normativen Prinzipien einhergeht mit einer signifikant geringeren Intensität der generellen Systemunterstützung in Ostdeutschland. Von einer einheitlichen politischen Kultur in diesem Bereich oder zumindest von einer im Zeitverlauf wachsenden Kongruenz der Orientierungsmuster in Ost- und Westdeutschland kann somit nicht gesprochen werden.

\section{Die Entwicklung der Einstellungen zur spezifischen Form der Demokratie in Deutschland und zu ihrem Funktionieren}

Die signifikanten Ost-West-Unterschiede in den Bevölkerungsorientierungen zeigen sich auch hinsichtlich der zweiten Objektebene der demokratischen Ordnung, der Strukturebene, die sich auf die durch die jeweilige Verfassung eines Staates festgelegte spezifische Form von Demokratie bezieht. Nach der Zufriedenheit mit der durch das Grundgesetz geformten Demokratie gefragt, bekundeten 2008 gut drei Viertel der Westdeutschen, aber weniger als drei Fünftel der Ostdeutschen ihre Zustimmung (vgl. Tabelle 3). Noch deutlicher werden die Ost-West-Unterschiede, wenn die spezifische Form der bundesrepublikanischen Demokratie im Vergleich mit anderen Staatsformen bewertet werden soll (vgl. Abbildung 2). Hier unterscheiden sich die Ost- und Westdeutschen nicht nur graduell, sondern prinzipiell voneinander.

Im Schnitt halten fast drei Viertel der Westdeutschen die im Grundgesetz festgelegte konkrete politische Ordnung für die beste Staatsform, und nur etwa jeder Zehnte ist der Auffassung, es gäbe eine andere Staatsform, die besser sei. ${ }^{27}$ In Ostdeutschland dagegen

27 Der Fragetext in Abbildung 2 lautet: „Glauben Sie, die Demokratie, die wir in der Bundesrepublik haben, ist die beste Staatsform, oder gibt es eine andere Staatsform, die besser ist?" 


\section{Tabelle 3: Einstellungen zur Demokratie des Grundgesetzes 2008 (Angaben in Prozent)}

„Nun geht es um die Demokratie in der Bundesrepublik. Was würden Sie allgemein zu der Demokratie in der Bundesrepublik Deutschland, also zu unserem ganzen politischen System sagen, so wie es in der Verfassung festgelegt ist? Sind Sie damit ...?"

\begin{tabular}{|l|ccc|}
\hline & Gesamt & Ost & West \\
\hline Sehr zufrieden & 19 & 10 & 21 \\
Eher zufrieden & 53 & 48 & 55 \\
Eher unzufrieden & 20 & 29 & 18 \\
Sehr unzufrieden & 5 & 8 & 4 \\
\hline Zu & &
\end{tabular}

Zu 100 Prozent fehlende Werte: weiß nicht / keine Antwort; $N=5681$.

Quelle: DPLG-Projekt „Demokratische und rechtsextreme Bevölkerungseinstellungen“, a.a.O. (Fn. 9).

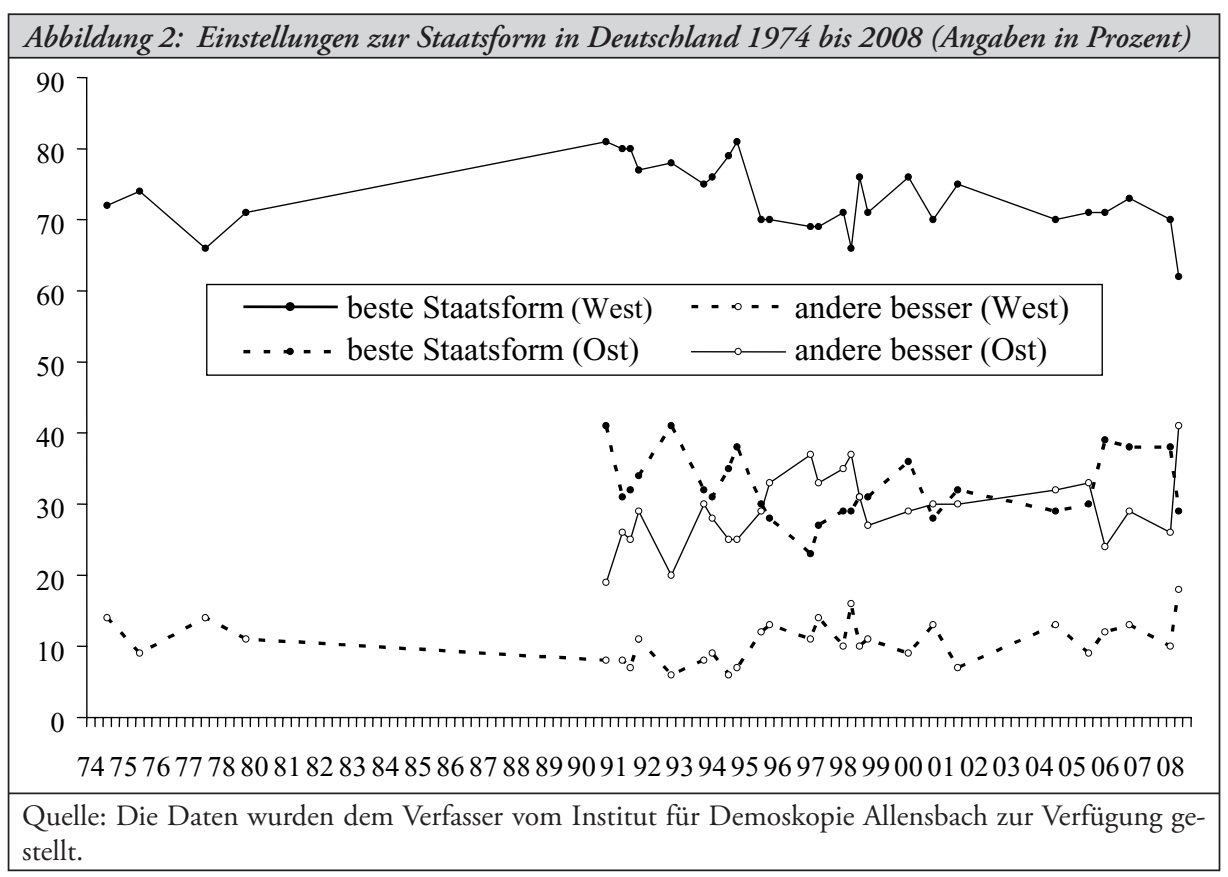

sprachen sich zu keiner Zeit mehr als zwei Fünftel für die bundesrepublikanische Demokratie als beste Staatsform aus, und seit Mitte der 1990er Jahre wechseln in den Befragungen die relativen Mehrheiten zwischen dieser Gruppe und denjenigen, die eine andere Staatsform für besser halten, wobei sich mehr als ein Drittel der Bürger in der Beurteilung unentschlossen zeigt.

Die Leistungsebene als dritte Objektebene der Orientierungen gegenüber der demokratischen politischen Ordnung bezieht sich auf ihr Funktionieren, das heißt die Verfassungswirklichkeit. Diese Orientierungsebene stellt auf die Wahrnehmung und Bewertung der Systemleistung ab, wird also im Positiven wie im Negativen durch den alltäglichen politischen Prozess bestimmt, so dass die Orientierungen in der Regel stärker schwanken.

So ist es sicherlich auch der beginnenden Einigungseuphorie zuzuschreiben, dass sich die Zufriedenheit mit dem Funktionieren der Demokratie in Westdeutschland in der ersten 
Hälfte des Jahres 1990 mit 81 Prozent auf dem höchsten jemals gemessenen Niveau befand (vgl. Abbildung 3). Da „das politische System der Bundesrepublik seit der Vereinigung unbestreitbar einer ernsten Belastungsprobe unterworfen " 28 war, verwundert es auch nicht, dass die Zufriedenheit der Westdeutschen mit dem Funktionieren der Demokratie nach dem Abklingen der Vereinigungseuphorie die hohen Werte der Zeit vor der Vereinigung wo im Schnitt etwa drei Viertel der Bürger mit der Systemleistung zufrieden waren - nicht mehr erreichte: Das Niveau ging in den ersten Jahren deutlich zurück, und man sprach für diesen Zeitraum sogar von der „schwerste(n) Akzeptanzkrise des politischen Systems seit dem Beginn der 70er Jahre"29. Danach erholte sich das Vertrauen in das Funktionieren der Demokratie wieder, stürzte jedoch in der Phase vor dem Regierungswechsel 1998 noch tiefer $\mathrm{ab}$, und in der Folgezeit setzten sich die zyklischen Schwankungen fort.

Betrachtet man den Untersuchungszeitraum nach dem Abklingen der Vereinigungseuphorie, so ist über die Schwankungen hinweg kein eindeutiger negativer oder positiver Trend festzustellen. Dies gilt auch für die Zufriedenheitswerte in Ostdeutschland, die allerdings deutlich unter denen des Westens liegen: Im Schnitt waren in den letzten eineinhalb Jahrzehnten etwa drei Fünftel der Westdeutschen und nur etwa zwei Fünftel der Ostdeutschen mit dem Funktionieren der Demokratie zufrieden. Zudem gibt es keinerlei Anzeichen für eine Verringerung des West-Ost-Gefälles. Dies bedeutet nicht nur einen quantitativen, sondern auch einen qualitativen Unterschied, da die Systemleistung über fast den gesamten Zeitraum hinweg von der absoluten Mehrheit der Westdeutschen positiv beurteilt wurde, während dies in Ostdeutschland niemals der Fall war.

Die differenzierten Daten von 2008 zeigen, dass nur sehr wenige Bürger mit dem Funktionieren der Demokratie „sehr zufrieden“ sind (vgl. Tabelle 4).

Während mit der durch das Grundgesetz festgelegten bundesrepublikanischen Form der Demokratie, also der Verfassungsnorm, noch jeder Fünfte sehr zufrieden ist (vgl. Tabelle 3), gilt dies hinsichtlich der Verfassungswirklichkeit nur noch für jeden Fünfundzwanzigsten. Nimmt man die „eher Zufriedenen“ hinzu, so ist in Westdeutschland knapp die Hälfte der Bürger mit dem Funktionieren der Demokratie zufrieden, in Ostdeutschland trifft dies nur für ein knappes Drittel zu.

$\mathrm{Zu}$ der in Deutschland implementierten, mit dem westdeutschen Demokratiemodell vor der Vereinigung identischen Form der Demokratie und ihrem Funktionieren hat somit ein großer Teil der Ostdeutschen auch fast zwei Jahrzehnte nach der Vereinigung noch „ein gebrochenes Verhältnis “30, und es gibt keine Anzeichen dafür, dass sich dies in naher $\mathrm{Zu}$ kunft ändern könnte.

28 Oscar W. Gabriel / Angelika Vetter, Politische Involvierung und politische Unterstützung im vereinigten Deutschland - Eine Zwischenbilanz, in: Fritz Plasser / Oscar W. Gabriel / Jürgen W. Falter / Peter A. Ulram (Hrsg.), Wahlen und politische Einstellungen in Deutschland und Österreich, Frankfurt am Main 1999, S. 191 - 240, S. 214.

29 Oscar W. Gabriel, Demokratie in der Vereinigungskrise? Struktur, Entwicklung und Bestimmungsfaktoren der Einstellungen zur Demokratie im vereinigten Deutschland, in: Zeitschrift für Politikwissenschaft, 9. Jg. (1999), H. 3, S. $827-861$, S. 836.

30 Oscar W. Gabriel, Bürger und Demokratie im vereinigten Deutschland, in: PVS, 48. Jg. (2007), H. 3, S. $540-552$, S. 546. 


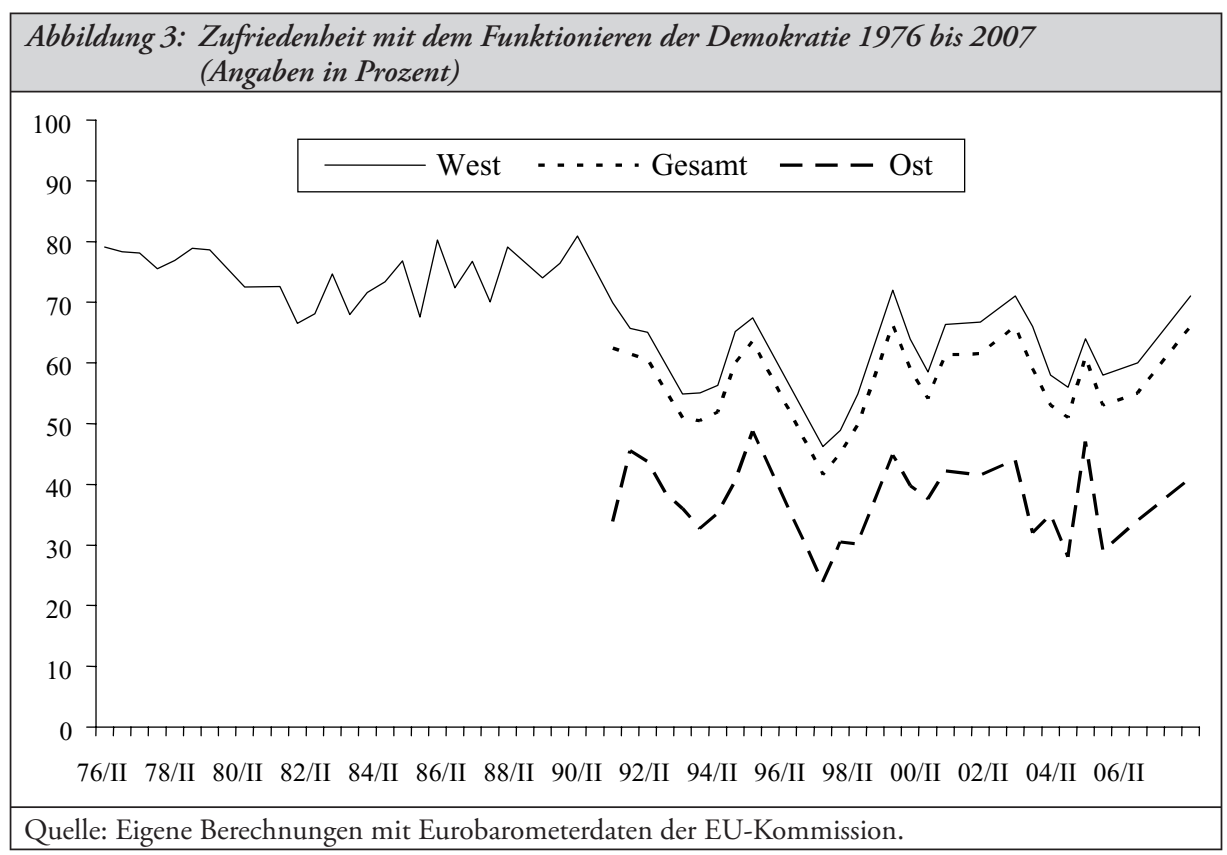

\section{Tabelle 4: Einstellungen zum tatsächlichen Funktionieren der Demokratie 2008 (Angaben in Prozent)}

„Und was würden Sie allgemein zu der Demokratie in der Bundesrepublik Deutschland, also zu unserem ganzen politischen System sagen, so wie es tatsächlich funktioniert? Sind Sie damit ...?"

\begin{tabular}{|l|crc|}
\hline & Gesamt & Ost & West \\
\hline Sehr zufrieden & 4 & 2 & 4 \\
Eher zufrieden & 40 & 30 & 43 \\
Eher unzufrieden & 44 & 52 & 42 \\
Sehr unzufrieden & 11 & 15 & 10 \\
\hline Zut
\end{tabular}

Zu 100 Prozent fehlende Werte: weiß nicht / keine Antwort; N = 5618 .

Quelle: DPLG-Projekt „Demokratische und rechtsextreme Bevölkerungseinstellungen“, a.a.O. (Fn. 9).

\section{Die Typologie der Einstellungen zur Demokratie und die Einflussfaktoren auf die Demokratieeinstellungen}

Für die Beurteilung des Verhältnisses der Bevölkerung zur demokratischen Ordnung genügt es jedoch nicht, die Haltung gegenüber den einzelnen Objektebenen getrennt zu betrachten. Ebenso wichtig ist die Analyse von Überzeugungssystemen, die sich aus der Kombination der individuellen Orientierungen gegenüber den drei Objektebenen ergeben. Im ersten Abschnitt wurde daher eine Typologie der Einstellungen zur demokratischen Ordnung vorgestellt, die zufriedene, politikkritische und systemkritische Demokraten sowie Antidemokraten unterscheidet.

Tabelle 5 zeigt die Verteilung dieser vier Typen im Jahre 2008. Zunächst wird deutlich, dass die Befragten durchaus in der Lage sind, die drei Objektebenen der Orientierungen 
gegenüber der demokratischen Ordnung analytisch zu trennen und auf diesbezügliche Fragen in einer konsistenten Weise zu antworten, wie der geringe Anteil von Nichtklassifizierbaren zeigt: 95 Prozent der Befragten besitzen ein in sich widerspruchsfreies Überzeugungssystem. ${ }^{31}$

\begin{tabular}{|l|ccc|}
\hline Tabelle 5: Typologie der Einstellungen zur Demokratie 2008 (Angaben in Prozent) \\
\hline & Gesamt & Ost & West \\
\hline Antidemokraten & 3 & 6 & 3 \\
Systemkritische Demokraten & 17 & 27 & 15 \\
Politikkritische Demokraten & 31 & 30 & 31 \\
Zufriedene Demokraten & 39 & 26 & 42 \\
Nicht klassifizierbar & 5 & 5 & 5 \\
\hline Zu 100 Prozent fehlende Werte: weiß nicht / keine Antwort; N = 5681. \\
Quelle: DPLG-Projekt „Demokratische und rechtsextreme Bevölkerungseinstellungen“, a.a.O. (Fn. 9). \\
\hline \multicolumn{4}{|l}{}
\end{tabular}

Sowohl in West- als auch in Ostdeutschland weist nur eine sehr kleine Minderheit ein konsistentes antidemokratisches Überzeugungssystem auf, allerdings ist diese Gruppe im Osten doppelt so groß wie im Westen. Auch systemkritische Demokraten, die der Idee der Demokratie positiv gegenüberstehen, aber sowohl mit der im Grundgesetz festgelegten spezifischen Form der Demokratie als auch mit ihrem tatsächlichen Funktionieren unzufrieden sind, gibt es im Osten der Republik fast doppelt so viele wie im Westen. Der Anteil der politikkritischen Demokraten, deren Kritik sich nur auf die Verfassungswirklichkeit richtet, ist gleich. Zur Gruppe der zufriedenen Demokraten zählen im Westen dagegen mehr als zwei Fünftel, in Ostdeutschland jedoch nur gut ein Viertel der Bürger. Insgesamt bestehen somit in Ostdeutschland hinsichtlich der Orientierungen gegenüber der Werte- und Strukturebene der Demokratie deutlich kritischere Überzeugungssysteme als im Westen.

Über die Gründe für die unterschiedlichen Orientierungsmuster in West und Ost besteht in der Forschung keine Einigkeit, da „bisher keine umfassende, empirisch bewährte Theorie demokratischer Orientierungen existiert" 32 . Die verschiedenen theoretischen Erklärungsstränge lassen sich vier Bereichen zuordnen ${ }^{33}$, die hier mit zentralen Thesen verknüpft werden sollen, die sich aus den Ansätzen ableiten lassen: Erstens der institutionenund akteursbezogene Ansatz mit der These: Je positiver die Bewertung der politischen Institutionen und Akteure ausfällt, desto positiver sind die Einstellungen zur demokratischen politischen Ordnung; zweitens der Modernisierungsansatz mit der These: Je stärker ein Individuum in das politische Geschehen involviert ist und je besser seine Ausstattung mit Ressourcen ist, desto positiver ist seine Einstellung zur Demokratie; drittens der Performanzansatz mit der These: Je besser die Leistung des politischen Systems in Bezug auf die individuelle Lebenssituation wahrgenommen wird, desto positiver sind die Einstellungen zur demokratischen politischen Ordnung; viertens der Sozialisationsansatz mit der These:

31 Zur Berechnung der Typologie wurden die Antworten der Befragten hinsichtlich der drei Objektebenen jeweils dichotomisiert, das heißt es wurde nur zwischen dafür/dagegen beziehungsweise zufrieden/nicht zufrieden unterschieden. Als nichtklassifizierbar wurden alle Befragten eingestuft, deren Antwortmuster nicht einem der vier Orientierungstypen entsprach.

32 Kerstin Völkl, a.a.O. (Fn. 12), S. 266.

33 Vgl. Kerstin Völkl, a.a.O. (Fn. 12), S. 266 ff. und Oscar W. Gabriel, a.a.O. (Fn. 23). 
Für Individuen, die in einem anderen, undemokratischen politischen System sozialisiert wurden, gilt, dass sie umso negativer die demokratische Ordnung beurteilen, je positiver ihre Einstellungen zu der zuvor bestehenden politischen Ordnung sind.

Die früheren getrennten Analysen der Beziehungen zwischen den verschiedenen Erklärungsfaktoren und den Einstellungen gegenüber der Werteebene und der Performanzebene der demokratischen Ordnung haben allerdings teilweise unterschiedliche Ergebnisse geliefert. Diese Analysen hatten jedoch das Problem, dass sie Personen mit unterschiedlichen Einstellungen jeweils in einer Gruppe zusammengefasst haben: Analysiert man die Beziehung zwischen Befürwortung / Ablehnung der Idee der Demokratie und verschiedenen Erklärungsfaktoren, so wirft man bei den Befürwortern die systemkritischen und politikkritischen Demokraten zusammen mit den zufriedenen Demokraten in einen Topf, analysiert man die Beziehung zwischen der Zufriedenheit / Unzufriedenheit mit dem Funktionieren der Demokratie und verschiedenen Erklärungsfaktoren, so behandelt man bei den Unzufriedenen die politikkritischen und systemkritischen Demokraten zusammen mit den Antidemokraten als eine Gruppe. Die hier vorgestellte Typologie vermeidet dies. Sie sollte auch nicht als ordinale Skala mit von Gruppe zu Gruppe kritischerer Einstellung zur demokratischen Ordnung im Allgemeinen betrachtet werden, sondern als Nominalskala, da sich die Einstellungen der verschiedenen Gruppen auf unterschiedliche Ebenen der Demokratie beziehen. So macht es die Typologie möglich zu analysieren, wie sich bestimmte Erklärungsfaktoren auf die jeweilige Größe der unterschiedlichen Typen demokratischer Überzeugungssysteme auswirken. Dies kann im Folgenden aus Platzmangel nur kursorisch geschehen, wobei die Gruppe der Antidemokraten im Mittelpunkt stehen soll. ${ }^{34}$

Die zuvor getroffenen Thesen lassen sich mit bestimmten Variablen aus unseren Umfragen von 2008 empirisch überprüfen. Die Daten der Berlin-Brandenburg-Studie zeigen, dass die Einstellungen zur Demokratie im Sinne des institutionen- und akteursbezogenen Ansatzes von den Einstellungen zu den Parteien abhängen. Unter denjenigen, die alle relevanten Parteien positiv beurteilen, gibt es keine Antidemokraten; unter denjenigen, die keine der Parteien positiv sehen, ist dagegen jeder Zwölfte ein Antidemokrat, und zwei Fünftel gehören zu den systemkritischen Demokraten.

Auch die beiden Faktoren, die sich dem modernisierungstheoretischen Ansatz zurechnen lassen, üben einen Einfluss auf die Art der Einstellung zur Demokratie aus. Bei den Befragten aus Berlin und Brandenburg besteht ein deutlicher Zusammenhang mit dem politischen Interesse: Unter denjenigen mit sehr starkem politischen Interesse gehört nur jeder Fünfzigste, unter denjenigen, die überhaupt nicht an Politik interessiert sind, jedoch jeder Zehnte zu den Antidemokraten, und zusätzlich zählen zwei Fünftel zu den systemkritischen Demokraten. Der Einfluss der individuellen Ressourcenausstattung lässt sich für Gesamtdeutschland zeigen: Betrachtet man die berufliche Stellung der Befragten, so sind die Beamten am wenigsten und die Arbeitslosen am stärksten demokratiekritisch eingestellt, und unter den Befragten mit niedrigem sozio-ökonomischen Status, dass heißt niedriger Bildung und niedrigem Einkommen, ist jeder Zehnte ein Antidemokrat, während unter den Befragten mit hohem Status kaum Antidemokraten zu finden sind.

Auch von sozialen Ungerechtigkeits- und Benachteiligungsgefühlen, also einer negativen Beurteilung der Leistung des politischen Systems, wird die Art der demokratischen Einstel-

$34 \mathrm{Zu}$ den Ergebnissen vgl. im Einzelnen Oskar Niedermayer / Richard Stöss, a.a.O. (Fn. 9). 
lungen deutlich beeinflusst. Unter den Berlinern und Brandenburgern, die der Ansicht sind, dass es in unserer Gesellschaftsordnung im Großen und Ganzen eher gerecht zugeht, gibt es keine Antidemokraten und fast zwei Drittel zufriedene Demokraten; unter denjenigen, die die Gesellschaftsordnung eher für ungerecht halten, gehört jedoch jeder Fünfzehnte zu den Antidemokraten und nur jeder Sechste zu den zufriedenen Demokraten. Bei den Befragten, die sich zu den Benachteiligten zählen, finden sich dreimal so viele Antidemokraten wie bei denjenigen, die sich eher zu den Bevorzugten rechnen.

Für die These des Sozialisationsansatzes lässt sich anführen, dass die in der DDR sozialisierten Befragten aus der Region Berlin-Brandenburg, die noch eine Bindung an das alte Regime aufweisen, gegenüber der bundesrepublikanischen Form der Demokratie deutlich kritischer eingestellt sind als Befragte ohne eine solche Bindung.

Bedenkt man, dass in Ostdeutschland die Arbeitslosigkeit und der Anteil von Bürgern mit niedrigem sozio-ökonomischen Status deutlich höher ist als im Westen, soziale Ungerechtigkeits- beziehungsweise Benachteiligungsgefühle deutlich stärker verbreitet sind als im Westen und ein nicht zu vernachlässigender Teil der Ostdeutschen immer noch eine Bindung an das DDR-Regime aufweist, helfen diese Ergebnisse auch, die Ost-West-Unterschiede in den Überzeugungssystemen der Bürger zu erklären. Ein umfassendes Erklärungsmodell der Orientierungen gegenüber der demokratischen Ordnung unter Berücksichtigung sowohl der drei Objektebenen als auch aller relevanten Variablen der vier Erklärungsansätze, die sich ja nicht widersprechen, sondern eher ergänzen, kann jedoch auch hier nicht geliefert werden.

\section{Fazit: (Noch) keine Gefährdung der Demokratie}

Bei der Bewertung der Ergebnisse darf nicht vergessen werden, dass eine demokratische politische Ordnung - im Gegensatz zu allen anderen Herrschaftsordnungen - Kritik und Opposition zulässt und ihren Bürgern das Recht einräumt, ihre Unzufriedenheit in vielfältiger Form zum Ausdruck zu bringen. Insbesondere ist die Kritik an den realen politischen Verhältnissen beziehungsweise der jeweiligen konkreten Demokratiekonzeption eines politischen Systems nicht von vornherein als antidemokratisch zu werten, denn sie kann auch von demokratischen Wertvorstellungen getragen sein und als Antriebskraft demokratischer Reformen dienen. Das einer Demokratie angemessene Muster von Orientierungen gegenüber der politischen Ordnung besteht daher aus einer Verbindung von Loyalität und Kritikbereitschaft. Wenn allerdings nur noch eine Minderheit der Bürger mit der politischen Ordnung rundherum zufrieden ist, wirft dies schon die Frage auf, ob nicht Defizite in der Legitimation dieser Ordnung bestehen.

Zudem wurde deutlich, dass auch fast zwei Jahrzehnte nach der Wiedervereinigung von der Herausbildung eines demokratischen Grundkonsenses zwischen Ost- und Westdeutschen noch nicht gesprochen werden kann. Zwar akzeptieren die Bürger in Ostdeutschland die Idee der Demokratie als generelles Ordnungsmodell, aber die Intensität der Befürwortung und die Zustimmung zu den meisten essenziellen Merkmalen einer Demokratie ist signifikant geringer als im Westen, und noch deutlichere Unterschiede zeigen sich in den Orientierungen gegenüber der spezifischen bundesrepublikanischen Form der Demokratie und ihrem Funktionieren. 
Folgt man der Argumentation der traditionellen Political-Culture-Forschung ${ }^{35}$, ist eine solche Koexistenz zweier unterschiedlicher politischer Kulturen ein Indiz für die Inkon-gruenz von politischer Kultur und politischer Struktur, die längerfristig die Stabilität und Leistungsfähigkeit des politischen Systems gefährden kann. Dem ist jedoch zu entgegnen: „Demokratiekritische Einstellungen bei einem Teil der Bevölkerung reichen, für sich genommen, noch nicht aus, um eine demokratische politische Ordnung zu destabilisieren." 36 Das mit solcher Inkongruenz verbundene Problem liegt daher „nicht primär in den Auswirkungen dieses Zustandes auf die Performanz oder die Stabilität des politischen Systems. Politische Einstellungen beeinflussen das Funktionieren des politischen Systems nur dann, wenn kollektive Akteure sie artikulieren und als Basis politischer Mobilisierungsprozesse nutzen “37. Solange die Umsetzung von demokratiekritischen Überzeugungssystemen in antisystemisches kollektives Verhalten breiter Bevölkerungskreise nicht erfolgt, verbleibt die geschilderte Inkongruenz daher auf der normativen Ebene, das heißt sie stellt ein Legitimationsproblem des deutschen politischen Systems dar.

35 Vgl. hierzu grundlegend Gabriel A. Almond/ Sidney Verba, The Civic Culture, Boston 1965.

36 Max Kaase I Petra Bauer-Kaase, Deutsche Vereinigung und innere Einheit 1990-1997, in: Heiner Meulemann (Hrsg.), Werte und nationale Identität im vereinigten Deutschland - Erklärungsansätze der Umfrageforschung, Opladen 1998, S. 251 - 267.

37 Oscar W. Gabriel, a.a.O. (Fn. 30), S. 541.

\title{
Vertrauen und Kontrolle: Der Einfluss von Werten auf Einstellungen zu politischen Institutionen
}

\author{
Joachim Behnke
}

„If men were angels, no government would be necessary. If angels were to govern men, neither external nor internal controls on government would be necessary." ${ }^{1}$

Moderne Demokratien sind überhaupt nur noch in der Form repräsentativer Demokratie vorstellbar. Im Kern jeder Demokratietheorie steht daher das Verhältnis von Repräsentant zu Repräsentiertem, das seit John Locke üblicherweise mit dem Begriff des „Trusteeship“ bezeichnet wird. Diese Beziehung ähnelt formal, das heißt in ihrer Struktur als Versprechen $^{2}$, zwar einer einfachen Vertrauensbeziehung wie sie zum Beispiel auch bei Handelsgeschäften vorzufinden ist, aber gleichzeitig unterscheidet sie sich von dieser in einer entscheidenden Hinsicht: Der Bürger kann zwar wählen, wem er sein Vertrauen schenkt, aber wegen der Unentrinnbarkeit der formalen Struktur der Repräsentation kann er sich nicht entscheiden, sich überhaupt nicht repräsentieren zu lassen, das heißt er ist gewissermaßen zur Vertrauensvergabe verdammt, oder - genauer noch: Er ist in einer Situation gefangen,

1 Alexander Hamilton / James Madison / John Jay, The Federalist Papers, New York 1982 [1788], S. 262.

2 Vgl. James S. Coleman, Foundations of Social Theory, Cambridge 1990, S. 91 ff.; Bernd Lahno, Versprechen, München 1995. 